\title{
Some results on strong convergence for nonlinear maps in Banach spaces
}

\author{
Abdul Latif ${ }^{\mathrm{a}}$, Adnan Salem Alhomaidan ${ }^{\mathrm{a}}$, Xiaolong Qin ${ }^{\mathrm{b}, *}$ \\ ${ }^{a}$ Department of Mathematics, King Abdulaziz University, P. O. Box 80203, Jeddah-21589, Saudi Arabia. \\ ${ }^{b}$ Institute of Fundamental and Frontier Sciences, University of Electronic Science and Technology of China, Sichuan, China.
}

Communicated by Y. H. Yao

\begin{abstract}
In this paper, an equilibrium problem which is also known as the Ky Fan inequality is investigated based on a fixed point method. Strong convergence theorems for solutions of the equilibrium problem are established in the framework of reflexive Banach spaces. Applications are also provided to support the main results. (c)2017 All rights reserved.
\end{abstract}

Keywords: Equilibrium problem, fixed point, nonexpansive mapping, variational inequality, hybrid method. 2010 MSC: 47H09, 47N10, 90C30.

\section{Introduction}

Let $E$ be a real Banach space and let $C$ be a convex and closed subset of $E$. $\mathbb{R}$ stands for the set of real numbers. Let $B: C \times C \rightarrow \mathbb{R}$ be a function. In this paper, we concern with the following inequality, which was first studied by Ky Fan [10]. Find an $x \in C$ such that

$$
\mathrm{B}(x, y) \geqslant 0, \quad \forall y \in C .
$$

This inequality is called the Ky Fan inequality. It is also known as the equilibrium problem in the sense of Blum and Oettli [5]. In what follows, we use Sol(B) to denote the solution set of the Ky Fan inequality. The Ky Fan inequality, which includes many important problems in convex optimization and nonlinear functional analysis fields such as game theory, nonlinear complementarity problems, zero point problems, fixed point problems, and saddle point problems, recently has been extensively studied as a powerful and effective tool for solving problems which arise in the real world, for instance, economics, finance, transportation, ecology, and network; see $[2,7,8,11,17,19]$ and the references therein.

Mann-type iterative algorithms and Ishikawa-type iterative algorithms are efficient to approximate fixed points of nonlinear operators. However, they are only weakly convergent in infinite-dimensional Banach spaces. In many disciplines, including economics, image recovery, and quantum physics problems arise in infinite dimension spaces. In such problems, strong convergence is often much more desirable than weak convergence for it translates the physically tangible property that the energy $\left\|x_{n}-x\right\|$ of the

\footnotetext{
*Corresponding author

Email addresses: alatif@kau.edu.sa (Abdul Latif), qxlxajh@163.com (Xiaolong Qin)
} 
error between the iterate $x_{n}$ and the solution $x$ eventually becomes arbitrarily small. Recently, various regularization methods, in particular, projection methods, have been extensively investigated by many authors; see $[4,15]$ and the references therein.

In this paper, we propose a projection method for finding a common solution of an uncountable family of the Ky Fan inequalities. Strong convergence theorems of common solutions are established in the framework of real reflexive Banach spaces. The highlights of this paper are the framework of the space, which do not require the uniform smoothness, the uniform convexness and the parallel projection method which is efficient for an uncountable family of nonlinear operators. The paper is organized as follows. In Section 2, we provide some necessary definitions, properties and lemmas. In Section 3, the main strong convergence theorems are established in the framework of real reflexive Banach spaces. In Section 4, some applications are provided to support our main results.

\section{Preliminaries}

From now on, we use $E^{*}$ to stand for the dual space of $E$. Recall that the normalized duality mapping $\mathrm{J}$ from $\mathrm{E}$ to $2^{\mathrm{E}^{*}}$ is defined by

$$
\text { Jf }:=\left\{g^{*} \in E^{*}:\left\|g^{*}\right\|^{2}=\|f\|^{2}=\left\langle f, g^{*}\right\rangle\right\} .
$$

Let $B_{E}$ be the unit sphere of $E$. Recall that a Banach space $E$ is said to be strictly convex if and only if $\| x+$ $y \|<2$ for all $x, y \in B_{E}$ with $x \neq y$. E is said to be uniformly convex if and only if $\lim _{n \rightarrow \infty}\left\|u_{n}-v_{n}\right\|=0$, where $\left\{u_{n}\right\}$ and $\left\{v_{n}\right\}$ in $B_{E}$ and $\lim _{n \rightarrow \infty}\left\|u_{n}+v_{n}\right\|=2$. E is said to have a Gateaux differentiable norm or smooth if and only if $\lim _{s \rightarrow \infty}(\|s x+y\|-s\|x\|)$ exists for all $x, y \in U_{E}$. $E$ is said to have a uniformly Gâteaux differentiable norm if for all $y \in B_{E}, \lim _{s \rightarrow \infty}(s\|x\|-\|s x+y\|)$ is uniformly obtained $\forall x \in B_{E}$. $E$ is said to be have a Fréchet differentiable norm if and only if for each $x \in B_{E}, \lim _{s \rightarrow \infty}(s\|x\|-\|s x+y\|)$ is attained uniformly for all $y \in B_{E}$. $E$ is said to be have a uniformly Fréchet differentiable norm if $\lim _{s \rightarrow \infty}(s\|x\|-\|s x+y\|)$ is attained uniformly for all $x, y \in B_{E}$.

It is known if $E$ is uniformly smooth, then $J$ is uniformly norm-to-norm continuous on every bounded subset of $E$; if $E$ is a smooth Banach space, then $J$ is single-valued and demicontinuous, i.e., continuous from the strong topology of $E$ to the weak star topology of $E$; if $E$ is a strictly convex Banach space, then $J$ is strictly monotone; if $E$ is a reflexive and strictly convex Banach space with a strictly convex dual $E^{*}$ and $\mathrm{J}^{*}: \mathrm{E}^{*} \rightarrow \mathrm{E}$ is the normalized duality mapping in $\mathrm{E}^{*}$, then $\mathrm{J}^{-1}=\mathrm{J}^{*}$; if $\mathrm{E}$ is a smooth, strictly convex, and reflexive Banach space, then $J$ is single-valued, one-to-one, and onto; if $E$ is a uniformly smooth, then it is smooth and reflexive. It is also known that $E^{*}$ is uniformly convex if and only if $E$ is uniformly smooth.

From now on, we use symbols $\rightarrow$ and $\rightarrow$ to denote the strong convergence and weak convergence, respectively. Recall that $E$ has the Kadec-Klee property (KKP) [9] if $x_{n} \rightarrow x$ and $\left\|x_{n}\right\| \rightarrow\|x\|$, then $\left\|x_{n}-x\right\| \rightarrow 0$ as $n \rightarrow \infty$, where $\left\{x_{n}\right\}$ is any sequence in $E$, and $x$ is a point in $E$. We also remark here that there exist uniformly convex Banach spaces which have neither the Opial's property nor the Fréchet differentiable norm but their duals have the KKP; see [12] and the references therein.

Example 2.1 ([12]). Let $E$ be the $L^{p}[0,1]$, where $1 \leqslant p<\infty$ but $p \neq 2$. Let $F$ be $\mathbb{R}^{2}$ with the standard norm. The Cartesian product of $E$ and $F$ furnished with the $l^{2}$-norm is a uniformly convex space, its norm is not Fréchet differentiable, and it also does not have the Opial's property. But its dual has the KKP.

Let $M$ be a mapping on $E$. In this paper, we use $F p(M)$ to stand for the fixed point set of $M$. Recall that a point $q$ is said to be an asymptotic fixed point of $M$ iff $E$ contains a sequence $x_{n} \rightarrow q$ such that $\left\|x_{n}-M x_{n}\right\| \rightarrow 0$ as $n \rightarrow \infty$. The set of asymptotic fixed points of $M$ is denoted by $\operatorname{Afp}(M)$ in this paper.

Let $E$ be a real smooth Banach space in which $J$ is single-valued. We investigate the functional which is defined by

$$
\phi(x, y):=\|x\|^{2}+\|y\|^{2}-2\langle x, J y\rangle, \quad \forall x, y \in E .
$$

Let $C$ be a convex and closed subset of a real Hilbert space $H$. For any $x \in H$, there exists a unique nearest point in $C$, denoted by $P_{C} x$, such that $\|x-y\| \geqslant\left\|x-P_{C} x\right\|$ for all $y \in C$. The operator $P_{C}$ is called 
the metric projection from $H$ onto $C$. It is known that $P_{C}$ is firmly nonexpansive, that is, $\left\|P_{C} x-P_{C} y\right\|^{2} \leqslant$ $\left\langle P_{C} x-P_{C} y, x-y\right\rangle$. In [3], a new operator Proj ${ }_{C}$ was introduced based on operator $P_{C}$ in the framework of Banach spaces. The generalized projection $\operatorname{Proj}_{C}: E \rightarrow C$ is a mapping that assigns to an arbitrary point $x \in E$ the minimum point of $\phi(x, y)$. From the definition, we have the following inequality

$$
\|x\|^{2}+\|y\|^{2}-2\langle x, J y\rangle=\phi(x, y) \geqslant(\|x\|-\|y\|)^{2}, \forall x, y \in E .
$$

Recall that a mapping $M$ is said to be relatively asymptotically nonexpansive [1] iff, $\forall q \in A f p(M)=$ $\mathrm{Fp}(M) \neq \emptyset, \forall \mathrm{p} \in \mathrm{E}, \forall \mathrm{n} \geqslant 1$,

$$
\phi\left(q, M^{n} p\right) \leqslant \phi(q, p)+\mu_{n} \phi(q, p),
$$

where $\left\{\mu_{n}\right\} \subset[0, \infty)$ is a sequence such that $\mu_{n} \rightarrow 0$ as $n \rightarrow \infty$.

$M$ is said to be relatively nonexpansive [6] iff, $\forall q \in A f p(M)=F p(M) \neq \emptyset, \forall p \in E$,

$$
\phi(q, M p) \leqslant \phi(q, p) .
$$

$M$ is said to be asymptotically quasi- $\phi$-nonexpansive [15] iff, $\forall q \in F p(M) \neq \emptyset, p \in E, \forall n \geqslant 1$,

$$
\phi\left(q, M^{n} x\right) \leqslant \phi(q, p)+\mu_{n} \phi(q, p),
$$

where $\left\{\mu_{n}\right\} \subset[0, \infty)$ is a sequence such that $\mu_{n} \rightarrow 0$ as $n \rightarrow \infty$.

$M$ is said to be quasi- $\phi$-nonexpansive [14] iff, $\forall q \in \operatorname{Afp}(M) \neq \emptyset, \forall p \in E$,

$$
\phi(q, M p) \leqslant \phi(q, p) \text {. }
$$

Remark 2.2. The class of quasi- $\phi$-nonexpansive mappings is more desirable than the class of relatively nonexpansive mappings. Quasi- $\phi$-nonexpansive mappings, which are reduced to quasi-nonexpansive mappings in the framework of Hilbert spaces $(\sqrt{\phi(x, y)}=\|x-y\|)$, do not require strong restriction $F p(M)=A f p(M)$; see [14] and the references therein.

The following conditions are essential in this paper for studying equilibrium problem (1.1).

(C-1) $\mathrm{B}(\mathrm{s}, \mathrm{s})=0, \forall \mathrm{s} \in \mathrm{C}$;

$(C-2) B(s, r) \geqslant \lim _{e \rightarrow 0^{+}} B((1-e) s+e t, r), \forall s, t, r \in C$;

(C-3) $0 \geqslant B(t, s)+B(s, t), \forall s, t \in C$;

(C-4) for each $s \in C, t \mapsto B(s, t)$ is weakly lower semi-continuous and convex.

In addition, we also need the following lemmas to obtain our main results.

Lemma 2.3 ([3]). Let $\mathrm{E}$ be a reflexive, strictly convex, and smooth Banach space. Let $\mathrm{C}$ be a convex and closed convex subset of $\mathrm{E}$. Let $\mathrm{s} \in \mathrm{E}$. Then

$$
\phi\left(t, \operatorname{Proj}_{C} s\right) \leqslant \phi(t, s)-\phi\left(\operatorname{Proj}_{C} s, s\right), \quad \forall t \in C .
$$

Lemma 2.4 ([3]). Let $\mathrm{E}$ be a smooth Banach space $\mathrm{E}$ and let $\mathrm{C}$ be a convex and closed subset of $\mathrm{E}$. Let $\mathrm{s}_{0} \in \mathrm{C}$ and $s \in \mathrm{E}$. Then $\inf \{\phi(\mathrm{t}, \mathrm{s}): z \in \mathrm{C}\}=\phi\left(\mathrm{s}_{0}, \mathrm{~s}\right)$ iff

$$
0 \leqslant\left\langle s_{0}-\mathrm{r}, \mathrm{J} s-\mathrm{J} s_{0}\right\rangle, \quad \forall \mathrm{r} \in \mathrm{C} .
$$

Lemma 2.5. Let $\mathrm{E}$ be a smooth, strictly convex and reflexive Banach space $\mathrm{E}$ and let $\mathrm{C}$ be a convex and closed subset of $\mathrm{E}$. Let $\mathrm{B}$ be a bifunction with (C-1)-(C-4). Let $\mathrm{r}>0$ and $\mathrm{x} \in \mathrm{E}$. Then

(a) there exists $v \in \mathrm{C}$ such that [5]

$$
\mathrm{rB}(v, \mathrm{t}) \geqslant\langle v-\mathrm{t}, \mathrm{J} v-\mathrm{J} s\rangle, \quad \forall \mathrm{t} \in \mathrm{C} ;
$$

(b) define a mapping $\mathrm{S}^{\mathrm{r}, \mathrm{B}}: \mathrm{E} \rightarrow \mathrm{C}$ by $([14,18])$

$$
\mathrm{S}^{r, \mathrm{~B}} \mathrm{~s}=\{v \in \mathrm{C}: \mathrm{rB}(v, \mathrm{t}) \geqslant\langle v-\mathrm{t}, \mathrm{J} v-\mathrm{Js}\rangle, \quad \forall \mathrm{t} \in \mathrm{C}\} .
$$

Then the following conclusions hold: 
(1) $\mathrm{Fp}\left(\mathrm{S}_{\mathrm{r}}\right)=\mathrm{Sol}(\mathrm{B})$;

(2) $S^{r, B}$ is quasi- $\phi$-nonexpansive and satisfies the inequality

$$
\phi\left(S^{r, B} x, x\right) \leqslant \phi(q, x)-\phi\left(q, S^{r, B} x\right), \forall q \in F p\left(S_{r}\right) .
$$

Remark 2.6. If $\mathrm{B}(\mathrm{s}, \mathrm{t}) \equiv 0$ for all $\mathrm{s}, \mathrm{t} \in \mathrm{C}$, then $\mathrm{S}^{\mathrm{r}, \mathrm{B}}$ is reduced to $\mathrm{P}_{\mathrm{C}}$, the metric projection, in the framework of Hilbert spaces.

Remark 2.7 ([14]). Let Proj ${ }_{C}$ be the generalized projection operator from a smooth, strictly convex, and reflexive Banach space $E$ onto a convex and closed subset $C$ of $E$. Then $\operatorname{Proj}_{C}$ is a closed and quasi- $\phi-$ nonexpansive mapping with $\mathrm{Fp}\left(\operatorname{Proj}_{\mathrm{C}}\right)=\mathrm{C}$.

Remark 2.8 ([14]). Let $E$ be a strictly convex, reflexive, and smooth Banach space, and $M$ is a maximal monotone mapping with a nonempty zero point set $M^{-1}(0)$. Then $J^{r, M}=(J+r M)^{-1} J: E \rightarrow D(M)$, where $D(M)$ denotes the domain of $M$, is a closed quasi- $\phi$-nonexpansive mapping with $A^{-1}(0)=F p\left(J^{r, M}\right)$, where $r>0$ is real number.

Example 2.9. Let $E$ be any smooth Banach space and define a mapping $S$ on $E$ by

$$
S x= \begin{cases}-x, & x \neq\left(\frac{1}{3}+\frac{1}{3^{n}}\right) x^{\prime}, \\ \left(\frac{1}{3^{n+1}}+\frac{1}{3}\right) x^{\prime}, & x=\left(\frac{1}{3}+\frac{1}{3^{n}}\right) x^{\prime},\end{cases}
$$

for $n=1,2,3 \cdots$, where $x^{\prime}$ is a nonzero element in $E$. Then $S$ is a quasi- $\phi$-nonexpansive mapping but not relatively nonexpansive mapping. From the definition, we see that $S$ has a unique fixed point 0 . Note that

$$
\frac{\|S x\|^{2}-\|x\|^{2}}{2} \leqslant\langle J S x-J x, 0\rangle=\langle J S x-J x, p\rangle .
$$

It follows that

$$
\frac{\|p\|^{2}}{2}-\langle p, J S x\rangle+\frac{\|S x\|^{2}}{2} \leqslant \frac{\|p\|^{2}}{2}-\langle p, J x\rangle+\frac{\|x\|^{2}}{2}
$$

for all $x \in E$, that is, $\phi(p, x) \geqslant \phi(p, S x)$. S is quasi- $\phi$-nonexpansive. Next, we prove that $T$ is not a relatively nonexpansive. Let

$$
x_{n}=\left(\frac{1}{3}+\frac{1}{3^{n}}\right) x^{\prime}
$$

Using the definition, we have

$$
\left(\frac{1}{3}+\frac{1}{3^{n+1}}\right) x^{\prime}=S x_{n}
$$

This implies $\lim _{m \rightarrow \infty}\left\|x_{m}-S x_{m}\right\|=0$ and $x_{m} \rightarrow x^{\prime}$ as $m \rightarrow \infty$. That is, $x^{\prime}$ is in $\operatorname{Afp}(S)$ but not in $\operatorname{Fp}(S)$.

\section{Main results}

Theorem 3.1. Let $\mathrm{E}$ be a strictly convex, smooth, and reflexive Banach space. Let $\Lambda$ be an index set and let $\mathrm{C}$ be a convex closed subset of $\mathrm{E}_{\text {. Let }} \mathrm{B}_{i}$ be a function with (C-1)-(C-4) for every $i \in \Lambda$. Assume that $\cap_{i \in \Lambda} \operatorname{Sol}\left(B_{i}\right)$ is not empty and both $\mathrm{E}$ and $\mathrm{E}^{*}$ have the KKP. Let $\left\{\mathrm{x}_{\mathrm{n}}\right\}$ be a sequence generated in the following algorithm: $\mathrm{x}_{0} \in \mathrm{E}$ is chosen arbitrarily,

$$
\left\{\begin{array}{l}
C_{(1, i)}=C, x_{1}=\operatorname{Proj}_{C_{1}:=\cap_{i \in \Lambda} C_{(1, i)}} x_{0}, \\
r_{(n, i)} B_{i}\left(u_{(n, i)}, y\right) \geqslant\left\langle u_{(n, i)}-y, J u_{(n, i)}-J x_{n}\right\rangle, \quad \forall y \in C_{n}, \\
C_{(n+1, i)}=\left\{\mu \in C_{(n, i)}: \phi\left(\mu, u_{(n, i)}\right) \leqslant \phi\left(\mu, x_{n}\right)\right\}, \\
x_{n+1}=\operatorname{Proj}_{C_{n+1}:=\cap_{i \in \Lambda} C_{(n+1, i)}} x_{1},
\end{array}\right.
$$

where $\left\{r_{(n, i)}\right\}$ is such that $\liminf _{n \rightarrow \infty} r_{(n, i)}>0$ for every $i \in \Lambda$. Then $\left\{x_{n}\right\}$ converges strongly to Proj $\operatorname{P~}_{\cap_{i \in \Lambda} \operatorname{Sol}\left(B_{i}\right)} x_{1}$. 
Proof. From Lemma 2.5, we see that $\operatorname{Sol}\left(B_{i}\right)$ is convex and closed for each $i \in \Lambda$. Hence, $\cap_{i \in \Lambda} \operatorname{Sol}\left(B_{i}\right)$ is convex and closed. Therefore, $\operatorname{Proj}_{\cap_{i \in \Lambda} \operatorname{Sol}\left(B_{i}\right)} x_{1}$ is well-defined. Assume that $C_{(h, i)}$ is convex and closed for some $h \geqslant 1$. Letting $\mu_{1}$ and $\mu_{2}$ be two elements in $C_{(h+1, i)}$, we get $\mu_{1}, \mu_{2} \in C_{(h, i)}$. It follows that $\mu \in C_{(h, i)}$, where $\mu=(1-t) \mu_{2}+t \mu_{1}, t \in(0,1)$. Notice that $\phi\left(\mu_{2}, x_{h}\right) \geqslant \phi\left(\mu_{2}, u_{(h, i)}\right)$, and $\phi\left(\mu_{1}, x_{h}\right) \geqslant$ $\phi\left(\mu_{1}, \mathfrak{u}_{(h, i)}\right)$. This implies

$$
\left\langle\mu_{2}, J x_{h}-J u_{(h, i)}\right\rangle+\frac{\left\|u_{(h, i)}\right\|^{2}}{2} \leqslant \frac{\left\|x_{h}\right\|^{2}}{2}
$$

and

$$
\left\langle\mu_{1}, J x_{h}-J u_{(h, i)}\right\rangle+\frac{\left\|u_{(h, i)}\right\|^{2}}{2} \leqslant \frac{\left\|x_{h}\right\|^{2}}{2} .
$$

Using the above relations, one has

$$
\left\langle\mu, J x_{h}-J u_{(h, i)}\right\rangle+\frac{\left\|u_{(h, i)}\right\|^{2}}{2} \leqslant \frac{\left\|x_{h}\right\|^{2}}{2} .
$$

Hence, we have $\phi\left(\mu, x_{h}\right) \geqslant \phi\left(\mu, u_{(h, i)}\right)$, where $\mu$ is in $C_{(h, i)}$. This finds that $C_{(h+1, i)}$ is convex and closed. So, $C_{(n, i)}$ is convex and closed. This proves the projection onto $C_{n}$ is well-defined.

Now, we are in a position to show that $\cap_{i \in \Lambda} \operatorname{Sol}\left(B_{i}\right)$ is a subset of $C_{n}$. Note that the common solution set is a subset of $C$, where $C=C_{1}$. Suppose that $\cap_{i \in \Lambda} \operatorname{Sol}\left(B_{i}\right)$ is a subset of $C_{(h, i)}$. For any $\mu \in \cap_{i \in \Lambda} \operatorname{Sol}\left(B_{i}\right)$, which is a subset of $C_{(h, i)}$, we see that

$$
\phi\left(\mu, x_{h}\right) \geqslant \phi\left(\mu, S^{r_{(h, i)}, B} \chi_{h}\right)=\phi\left(\mu, u_{(h, i)}\right),
$$

which finds $\mu \in C_{(h+1, i)}$. This implies that $\cap_{i \in \Lambda} \operatorname{Sol}\left(B_{i}\right)$ which is a subset of $C_{(h, i)}$. This in turn implies $\cap_{i \in \wedge} \operatorname{Sol}\left(B_{i}\right)$ is a subset of $C_{n}$. Using Lemma 2.4, one sees

$$
\left\langle\mu, J x_{1}-J x_{n}\right\rangle \leqslant\left\langle x_{n}, J x_{1}-J x_{n}\right\rangle
$$

for any $\mu \in C_{n}$. Since $\cap_{i \in \Lambda} \operatorname{Sol}\left(B_{i}\right)$ is a subset of $C_{n}$, we find

$$
\left\langle\mu-x_{n}, J x_{1}-J x_{n}\right\rangle \leqslant 0
$$

for all $\mu \in \cap_{i \in \Lambda} \operatorname{Sol}\left(B_{i}\right)$. It follows from Lemma 2.3 that

$$
\phi\left(\operatorname{Proj}_{\cap_{i \in \Lambda} \operatorname{Sol}\left(B_{i}\right)} x_{1}, x_{1}\right)-\phi\left(\operatorname{Proj}_{\cap_{i \in \Lambda} \operatorname{Sol}\left(B_{i}\right)} x_{1}, x_{n}\right) \geqslant \phi\left(x_{n}, x_{1}\right) .
$$

This shows

$$
0 \leqslant \phi\left(x_{n}, x_{1}\right) \leqslant \phi\left(\operatorname{Proj}_{\cap_{i} \in \Lambda} \operatorname{Sol}\left(B_{i}\right) x_{1}, x_{1}\right),
$$

which is an upper bound. Hence, $\left\{x_{n}\right\}$ is a bounded sequence in C. Since the framework of the space is reflexive, we may assume that $x_{n} \rightarrow \bar{x} \in C_{n}$. Note that

$$
\limsup _{n \rightarrow \infty} \phi\left(x_{n}, x_{1}\right) \geqslant \liminf _{n \rightarrow \infty}\left(\left\|x_{n}\right\|^{2}+\left\|x_{1}\right\|^{2}-2\left\langle x_{n}, J x_{1}\right\rangle\right) \geqslant \phi\left(\bar{x}, x_{1}\right) \geqslant 0 .
$$

Using the fact that $\phi\left(\bar{x}, x_{1}\right) \geqslant \phi\left(x_{n}, x_{1}\right)$, one has $\lim _{n \rightarrow \infty}\left\|x_{n}\right\|=\|\bar{x}\|$. Using the KKP of $E$, we find $x_{n} \rightarrow \bar{x}$ as $n \rightarrow \infty$. Since

$$
\phi\left(x_{n+1}, x_{1}\right) \geqslant \phi\left(x_{n}, x_{1}\right),
$$

one finds from its boundedness that $\lim _{n \rightarrow \infty} \phi\left(x_{n}, x_{1}\right)$ exists. It follows that

$$
\phi\left(x_{n+1}, \operatorname{Proj}_{C_{n}} x_{1}\right) \leqslant \phi\left(x_{n+1}, x_{1}\right)-\phi\left(x_{n}, x_{1}\right) .
$$

Hence, we have

$$
\lim _{n \rightarrow \infty} \phi\left(x_{n+1}, x_{n}\right)=0
$$


Since $x_{n+1}$ is in $C_{n+1}$, we find that $\phi\left(x_{n+1}, x_{n}\right) \geqslant \phi\left(x_{n+1}, u_{(n, i)}\right) \geqslant 0$. It follows that

$$
\lim _{n \rightarrow \infty} \phi\left(x_{n+1}, u_{(n, i)}\right)=0 \text {. }
$$

Therefore,

$$
\lim _{n \rightarrow \infty}\left(\left\|u_{(n, i)}\right\|-\left\|x_{n+1}\right\|\right)=0 .
$$

This implies that

$$
\lim _{n \rightarrow \infty}\left\|u_{(n, i)}\right\|=\|\bar{x}\|
$$

Hence, we have

$$
\lim _{n \rightarrow \infty}\left\|J u_{(n, i)}\right\|=\lim _{n \rightarrow \infty}\left\|\mathfrak{u}_{(n, i)}\right\|=\|\bar{x}\| .
$$

This means that $\left\{J u_{(n, i)}\right\}$ is bounded. Since both spaces $E$ and $E^{*}$ are reflexive, we may assume that $J u_{(n, i)} \rightarrow u^{(*, i)} \in E^{*}$. Using the reflexivity of space $E$, we find there exists an element $u^{i} \in E$ such that $\mathrm{Ju}^{i}=u^{(*, i)}$. It follows that

$$
\phi\left(x_{n+1}, u_{(n, i)}\right)+2\left\langle x_{n+1}, J u_{(n, i)}\right\rangle=\left\|J u_{(n, i)}\right\|^{2}+\left\|x_{n+1}\right\|^{2} .
$$

Taking $\lim \inf _{\mathrm{n} \rightarrow \infty}$ yields that

$$
0 \geqslant\|\bar{x}\|^{2}+\left\|u^{(*, i)}\right\|^{2}-2\left\langle\bar{x}, u^{(*, i)}\right\rangle=\|\bar{x}\|^{2}+\left\|u^{i}\right\|^{2}-2\left\langle\bar{x}, J u^{i}\right\rangle=\phi\left(\bar{x}, u^{i}\right) \geqslant 0,
$$

which shows that $\mathrm{J} \bar{\chi}=u^{(*, i)}$. Hence, $J u_{(n, i)} \rightarrow J \bar{x} \in E^{*}$. Using the fact that $E^{*}$ has the KKP, we obtain from (3.2) that $\lim _{n \rightarrow \infty} J u_{(n, i)}=J \bar{x}$. Hence, we have

$$
\lim _{n \rightarrow \infty}\left\|J x_{n}-J u_{(n, i)}\right\|=0 .
$$

Next, we show that $\bar{x}$ is indeed in $\cap_{i \in \Lambda} \operatorname{Sol}\left(B_{i}\right)$. Using the condition on $r_{(n, i)}$, we may assume, without loss of generality, that there exists a real positive number sequence $\left\{\lambda_{i}\right\}$ such that $r_{(n, i)} \geqslant \lambda_{i}>0$. It follows that

$$
\lim _{n \rightarrow \infty} \frac{\left\|J u_{(n, i)}-J x_{n}\right\|}{r_{(n, i)}}=0 .
$$

On the other hand, we have

$$
\left\langle y-u_{(n, i)}, J u_{(n, i)}-J x_{n}\right\rangle+r_{(n, i)} B_{i}\left(u_{(n, i)}, y\right) \geqslant 0, \quad \forall y \in C_{n}
$$

Therefore,

$$
\left\|y-u_{(n, i)}\right\|\left\|J u_{(n, i)}-J x_{n}\right\| \geqslant r_{(n, i)} B_{i}\left(y, u_{(n, i)}\right), \quad \forall y \in C_{n} .
$$

It follows from (3.3) that $B_{\mathfrak{i}}(y, \bar{x}) \leqslant 0, \forall y \in C_{n}$. For $0<t_{i}<1$, put

$$
y_{(t, i)}=t_{i} y+\left(1-t_{i}\right) \bar{x}
$$

It follows that $y_{(t, i)}$ is in $C_{n}$. Hence $B_{i}\left(\bar{x}, y_{(t, i)}\right) \geqslant 0$. It follows that

$$
t_{i} B_{i}\left(y_{(t, i)}, y\right) \geqslant t_{i} B_{i}\left(y_{(t, i)}, y\right)+\left(1-t_{i}\right) B_{i}\left(y_{(t, i)}, \bar{x}\right) \geqslant B_{i}\left(y_{(t, i)}, y_{(t, i)}\right) .
$$

Hence, $B_{i}(\bar{x}, y) \geqslant 0$. This shows that $\bar{x}$ is in $\operatorname{Sol}\left(B_{i}\right)$ for every $i \in \Lambda$. Hence, $\bar{x}$ is in $\cap_{i \in \Lambda} \operatorname{Sol}\left(B_{i}\right)$. It follows from (3.1) that

$$
\left\langle\bar{x}-z, J x_{1}-J \bar{x}\right\rangle \geqslant 0, \quad \forall z \in \cap_{i \in \Lambda} \operatorname{Sol}\left(B_{i}\right) .
$$

Using Lemma 2.3, we find that $\bar{x}=\operatorname{Proj}_{\cap_{i \in \Lambda} \operatorname{Sol}\left(B_{i}\right)} x_{1}$. This completes the proof.

For a single function, we find from Theorem 3.1 the following. 
Corollary 3.2. Let $\mathrm{E}$ be a strictly convex, reflexive, and smooth Banach space. Let $\mathrm{C}$ be a convex and closed subset of $\mathrm{E}$ and let $\mathrm{B}: \mathrm{C} \times \mathrm{C} \rightarrow \mathbb{R}$ be a function with $(\mathrm{C}-1)-(\mathrm{C}-2)$. Assume that $\mathrm{Sol}(\mathrm{B})$ is nonempty and both $\mathrm{E}$ and $\mathrm{E}^{*}$ have the KKP. Let $\left\{x_{n}\right\}$ be a sequence generated in the following algorithm: $x_{0} \in \mathrm{E}$ is chosen arbitrarily

$$
\left\{\begin{array}{l}
x_{1}=\operatorname{Proj}_{C_{1}:=C} x_{0}, \\
r_{n} B\left(u_{n}, y\right) \geqslant\left\langle u_{n}-y, J u_{n}-J x_{n}\right\rangle, \quad \forall y \in C_{n}, \\
C_{n+1}=\left\{\mu \in C_{n}: \phi\left(\mu, u_{n}\right) \leqslant \phi\left(\mu, x_{n}\right)\right\}, \\
x_{n+1}=\operatorname{Proj}_{C_{n+1}} x_{1},
\end{array}\right.
$$

where $\left\{r_{n}\right\}$ is a real sequence with $\liminf _{n \rightarrow \infty} r_{n}>0$. Then $\left\{x_{n}\right\}$ converges strongly to Proj $j_{\text {Sol }(B)} x_{1}$.

Remark 3.3. Theorem 3.1 improves Zhao's results [20] from a single function to an uncountable infinitely family of functions. And the algorithm is more efficient since $u_{(n, i)}$ is searched monotonicially in $C_{n}$ instead of always in C. Theorem 3.1 does not require that the framework of the space is both uniformly convex and uniformly smooth, which is a standard assumption in most of the related work. The typical example of the space in Theorem 3.1 is a strictly convex, reflexive and smooth Musielak-Orlicz space; see [13] and the references therein. In order to illustrate the effectiveness of the algorithm we give the following numerical results using software Matlab 7.0. Let $E$ be the set of real numbers and $C=[0,1.5]$. Let $S^{B_{1}}$ be $x \cdot \sin x$, which has a unique fixed point in $C$. If we choose $x_{0} \in C$ arbitrarily, then for 50 different initial values, we see all the results are convergent in Figure 1. Let $E$ be the set of real numbers and $C=[0,0.5]$. Let $S^{B_{2}}$ be $x \cdot \tan x$, which has a unique fixed point in $C$. If we choose $x_{0} \in C$ arbitrarily, then for 50 different initial values, we see all the results are convergent in Figure 2.

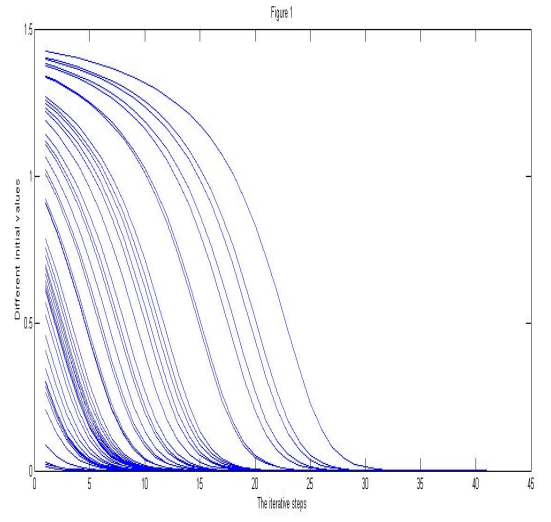

Figure 1

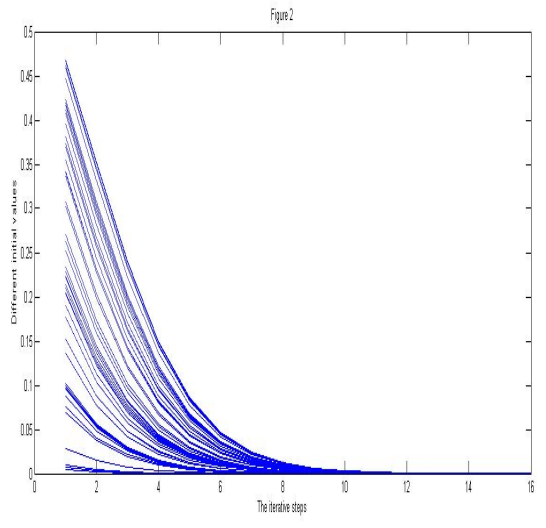

Figure 2

\section{Applications}

First, we consider a common solution problem of a family of variational inequalities. Let $A: C \rightarrow E^{*}$ be a single-valued monotone operator which is hemicontinuous (continuous along each line segment in $C$ with respect to the weak* topology of $\left.E^{*}\right)$. Consider the following variational inequality problem: find a point $x \in C$ such that $\langle x-y, A x\rangle \leqslant 0, \forall y \in C$. From now on, we use $\operatorname{Sol}(A)$ to stand for the solution set of the variational inequality and $\mathrm{Nc}(x)$ stands for the normal cone for $C$ at a point $x \in C$, $\operatorname{Nc}(x):=\left\{x^{*} \in E^{*}: 0 \geqslant\left\langle x^{*}, y-x\right\rangle, \forall y \in C\right\}$.

Theorem 4.1. Let $\mathrm{E}$ be a strictly convex, reflexive, and smooth Banach space. Let $\mathrm{C}$ be a convex and closed subset of $\mathrm{E}$ and let $\Lambda$ be an index set. Let $\mathrm{A}_{\mathrm{i}}: \mathrm{C} \rightarrow \mathrm{E}^{*}$ be a single-valued, monotone and hemicontinuous operator. Assume 
that $\cap_{i \in \Delta} \operatorname{Sol}\left(A_{i}\right)$ is not empty and both $\mathrm{E}$ and $\mathrm{E}^{*}$ have the KKP. Let $\left\{x_{n}\right\}$ be a sequence generated in the following algorithm

$$
\left\{\begin{array}{l}
x_{0} \in E, \text { chosen arbitrarily, } \\
C_{1, i}=C, x_{1}=\operatorname{Proj}_{C_{1}:=\cap_{i \in \Lambda} C_{1, i}} x_{0}, \\
u_{(n, i)}=\operatorname{Sol}\left(A_{i}+\frac{1}{r_{i}}\left(J-J x_{n}\right)\right), \\
C_{(n+1, i)}=\left\{u \in C_{(n, i)}: \phi\left(u, u_{(n, i)}\right) \leqslant \phi\left(u, x_{n}\right)\right\}, \\
C_{n+1}=\cap_{i \in \Lambda} C_{(n+1, i)}, x_{n+1}=\operatorname{Proj}_{C_{n+1}} x_{0}, \quad \forall n \geqslant 1,
\end{array}\right.
$$

where $r_{i}>0$ is a real number, $\forall i \in \Lambda$. Then $\left\{x_{n}\right\}$ converges strongly to $\operatorname{Proj}_{\cap_{i \in \Delta} \operatorname{Sol}\left(A_{i}\right)} x_{0}$.

Proof. For each $i \in \Lambda$, define a mapping $M_{i}$ by

$$
M_{i} x= \begin{cases}\emptyset, & x \notin C, \\ N_{c} x+A_{i} x, & x \in C .\end{cases}
$$

Then $M_{i}$ is a maximal monotone operator, and $\operatorname{Sol}\left(A_{i}\right)=M_{i}^{-1}(0)$; see Rockafellar [16]. For each $r_{i}>0$, and $x \in E$, there exists a unique $x_{r_{i}}$ in $D\left(M_{i}\right)$, where $D\left(M_{i}\right)$ denotes the domain of $M_{i}$, such that $J x \in r_{i} M_{i}\left(x_{r_{i}}\right)+J x_{r_{i}}$, where $x_{r_{i}}=\left(J+r_{i} M_{i}\right)^{-1} J x$. On the other hand, we have $z_{n, i}=\operatorname{Sol}\left(A_{i}+\frac{1}{r_{i}}\left(J-J x_{n}\right)\right)$, which is equivalent to $\frac{1}{r_{i}}\left(J x_{n}-J z_{n, i}\right) \in N_{C}\left(z_{n, i}\right)+A_{i} z_{n, i}$. This implies that $\left(J+r_{i} M_{i}\right)^{-1} J x_{n}=z_{n, i}$. Since $\left(J+r_{i} \partial M_{i}\right)^{-1} J$ is closed quasi- $\phi$-nonexpansive with $F p\left(\left(J+r_{i} \partial M_{i}\right)^{-1} J\right)=M_{i}^{-1}(0)[14]$ and using Theorem 3.1, we immediately find the desired conclusion.

Next, we study the problem of finding a common minimizer of a family of proper, lower semicontinuous, and convex functionals.

For a proper lower semicontinuous convex function $\mathrm{g}: \mathrm{E} \rightarrow(-\infty, \infty]$, the subdifferential mapping of $\mathrm{g}$ is defined by

$$
\partial g(x):=\left\{x^{*} \in E^{*}:\left\langle y-x, x^{*}\right\rangle \leqslant g(y)-g(x), \forall y \in E\right\}, \quad \forall x \in E .
$$

It is known [16] that the subdifferential mapping of $g$ is a maximal monotone operator and $0 \in \partial g(v) \Longleftrightarrow$ $\min _{x \in E} g(x)=g(v)$.

Theorem 4.2. Let $\mathrm{E}$ be a strictly convex, reflexive, and smooth Banach space. Let $\mathrm{C}$ be a convex and closed subset of $\mathrm{E}$ and let $\Lambda$ be an index set. Let $\mathrm{g}_{i}$ a proper, lower semicontinuous, and convex functional on $\mathrm{E}$ for every $i \in \Delta$. Assume that the common zero point set $\cap_{i \in \Lambda}\left(\partial g_{i}\right)^{-1}(0)$ is nonempty and both $\mathrm{E}$ and $\mathrm{E}^{*}$ have the KKP. Let $\left\{x_{n}\right\}$ be generated in the following algorithm:

$$
\left\{\begin{array}{l}
\mathrm{C}_{(1, i)}=\mathrm{C}, \mathrm{x}_{1}=\operatorname{Proj}_{\mathrm{C}_{1}:=\cap_{\mathrm{i} \in \Lambda} \mathrm{C}_{(1, i)} x_{0},} \\
\mathrm{u}_{(\mathrm{n}, \mathrm{i})}=\arg \min _{z \in E}\left\{2 r_{i} g_{i}(z)+\|z\|^{2}+\left\langle z, J x_{n}\right\rangle\right\}, \\
\mathrm{C}_{n+1, i}=\left\{u \in C_{n, i}: \phi\left(u, u_{(n, i)}\right) \leqslant \phi\left(u, x_{n}\right)\right\}, \\
\mathrm{C}_{n+1}=\cap_{i \in \Delta} C_{(n+1, i)}, x_{n+1}=\operatorname{Proj}_{C_{n+1}} x_{0}, \quad \forall n \geqslant 1,
\end{array}\right.
$$

where $r_{i}>0$ is a real number for all $i \in \Lambda$. Then $\left\{x_{n}\right\}$ converges strongly to $\Pi_{\cap_{i \in \Lambda}}\left(\partial g_{i}\right)^{-1}(0) x_{0}$.

Proof. For each $r_{i}>0$, and $x \in E$, we find that there exists a unique $x_{r_{i}}$ in $D\left(\partial g_{i}\right)$ such that $J x \in$ $r_{i} \partial g_{i}\left(x_{r_{i}}\right)+J x_{r_{i}}$, where $x_{r_{i}}=\left(J+r_{i} \partial g_{i}\right)^{-1} J x$.

$$
u_{(n, i)}=\arg \min _{z \in E}\left\{2\left\langle z, J x_{n}\right\rangle+2 r_{i} g_{i}(z)+\|z\|^{2}\right\}
$$

is equivalent to $0 \in \partial\left(g_{i}+\frac{\|\cdot\|^{2}}{2 r_{i}}+\frac{J x_{n}}{r_{i}}\right) u_{(n, i)}$. This finds that $u_{(n, i)}=\left(J+r_{i} \partial g_{i}\right)^{-1} J x_{n}$. Note that $(J+$ $\left.r_{i} \partial g_{i}\right)^{-1} J$ is closed quasi- $\phi$-nonexpansive with $F p\left(\left(J+r_{i} \partial g_{i}\right)^{-1} J\right)=\left(\partial g_{i}\right)^{-1}(0)$ [14]. Using Theorem 3.1, we immediately conclude the desired conclusion. 
Remark 4.3. In this paper, we studied a convex feasibility problem based on equilibrium problem (1.1) in the framework of Banach spaces and constructed a monotone projection algorithm for solving it. It deserves mentioning there is no restriction on the uniform smoothness or the uniform convexness. Our convergence analysis ensures that the proposed algorithm converges in norm to a special common solution that without any compact assumption imposed on the space or the bifunctions. We also apply the strong convergence result to variational inequality problems and convex minimization problems in the framework of Banach spaces.

\section{Acknowledgment}

This work was supported by the Deanship of Scientific Research (DSR), King Abdulaziz University, Jeddah, under grant No. D-020-130-1438. The authors, therefore, gratefully acknowledge the DSR technical and financial support.

\section{References}

[1] R. P. Agarwal, Y. J. Cho, X.-L. Qin, Generalized projection algorithms for nonlinear operators, Numer. Funct. Anal. Optim., 28 (2007), 1197-1215. 2

[2] R. Ahmad, M. Akram, H. A. Rizvi, Generalized f-vector equilibrium problem, Commun. Optim. Theory, 2014 (2014), 11 pages. 1

[3] Y. I. Alber, Metric and generalized projection operators in Banach spaces: properties and applications, Theory and applications of nonlinear operators of accretive and monotone type, Lecture Notes in Pure and Appl. Math., Dekker, New York, 178 (1996), 15-50. 2, 2.3, 2.4

[4] B. A. Bin Dehaish, X.-L. Qin, A. Latif, H. O. Bakodah, Weak and strong convergence of algorithms for the sum of two accretive operators with applications, J. Nonlinear Convex Anal., 16 (2015), 1321-1336. 1

[5] E. Blum, W. Oettli, From optimization and variational inequalities to equilibrium problems, Math. Student, 63 (1994), 123-145. 1, 2.5

[6] D. Butnariu, S. Reich, A. J. Zaslavski, Asymptotic behavior of relatively nonexpansive operators in Banach spaces, J. Appl. Anal., 7 (2001), 151-174. 2

[7] S. Y. Cho, B. A. Bin Dehaish, X.-L. Qin, Weak convergence of a splitting algorithm in Hilbert spaces, J. Appl. Anal. Comput., 7 (2017), 427-438. 1

[8] S. Y. Cho, X.-L. Qin, L. Wang, Strong convergence of a splitting algorithm for treating monotone operators, Fixed Point Theory Appl., 2014 (2014), 15 pages. 1

[9] I. Cioranescu, Geometry of Banach spaces, duality mappings and nonlinear problems, Mathematics and its Applications, Kluwer Academic Publishers Group, Dordrecht, (1990). 2

[10] K. Fan, A minimax inequality and applications, Inequalities, III (Proc. Third Sympos., Univ. California, Los Angeles, Calif., 1969; dedicated to the memory of Theodore S. Motzkin), Academic Press, New York, (1972), 103-113. 1

[11] N.-N. Fang, Some results on split variational inclusion and fixed point problems in Hilbert spaces, Commun. Optim. Theory, 2017 (2017), 13 pages. 1

[12] J. García Falset, W. Kaczor, T. Kuczumow, S. Reich, Weak convergence theorems for asymptotically nonexpansive mappings and semigroups, Nonlinear Anal., 43 (2001), 377-401. 2, 2.1

[13] H. Hudzik, W. Kowalewski, G. Lewicki, Approximate compactness and full rotundity in Musielak-Orlicz spaces and Lorentz-Orlicz spaces, Z. Anal. Anwend., 25 (2006), 163-192. 3.3

[14] X.-L. Qin, Y. J. Cho, S. M. Kang, Convergence theorems of common elements for equilibrium problems and fixed point problems in Banach spaces, J. Comput. Appl. Math., 225 (2009), 20-30. 2, 2.2, 2.5, 2.7, 2.8, 4, 4

[15] X.-L. Qin, Y. J. Cho, S. M. Kang, On hybrid projection methods for asymptotically quasi- $\phi$-nonexpansive mappings, Appl. Math. Comput., 215 (2010), 3874-3883. 1, 2

[16] R. T. Rockafellar, On the maximal monotonicity of subdifferential mappings, Pacific J. Math., 33 (1970), 209-216. 4

[17] T. V. Su, T. V. Dinh, On the existence of solutions of quasi-equilibrium problems (UPQEP), (LPQEP), (UWQEP) and (LWQEP) and related problems, Commun. Optim. Theory, 2016 (2016), 21 pages. 1

[18] W. Takahashi, K. Zembayashi, Strong and weak convergence theorems for equilibrium problems and relatively nonexpansive mappings in Banach spaces, Nonlinear Anal., 70 (2009), 45-57. 2.5

[19] Q.-N. Zhang, Nonlinear operators, equilibrium problems and monotone projection algorithms, Commun. Optim. Theory, 2017 (2017), 11 pages. 1

[20] J. Zhao, Approximation of solutions to an equilibrium problem in a nonuniformly smooth Banach space, J. Inequal. Appl., 2013 (2013), 10 pages. 3.3 